\title{
High-Energy Cosmic-Rays and Neutrinos around Supernova Shock Breakout
}

\section{Gwenael Giacinti*}

Max-Planck-Institut für Kernphysik, Postfach 103980, 69029 Heidelberg, Germany

E-mail: giacinti@mpi-hd.mpg.de

\begin{abstract}
Supernovae occurring in dense winds are promising candidates for particle acceleration to high energies. We focus here on the onset of particle acceleration, during the first couple of days following core-collapse. We show that a collisionless shock rapidly forms at supernova shock breakout. We calculate the CR energy that can be reached in such environments. We take the effect of cosmic-ray energy losses due to inelastic $p p$ and $p \gamma$ collisions into account, as well as possible damping of the turbulence by radiation. We find that protons are accelerated to multi$\mathrm{TeV}$ energies within minutes to hours for Wolf-Rayet and red supergiant progenitors. Secondary high-energy neutrinos with energies greater than $\sim 100 \mathrm{GeV}$ are expected to be produced.
\end{abstract}

35th International Cosmic Ray Conference - ICRC2017

10-20 July, 2017

Bexco, Busan, Korea

\footnotetext{
* Speaker.
} 


\section{Introduction}

Following core-collapse, a radiation-dominated shock (RDS) travels through the supernova progenitor. Once the RDS reaches the optically thin outer layers of the stellar core, or of the wind if optically thick, photons escape ahead of the shock. This flash of photons corresponds to shock breakout (SB), e.g. [1, 2, 3, 4, 5, 6, 7, 8, 9, 10, 11]. A collisionless shock (CS) later forms [12, 13]. The Larmor radius $r_{\mathrm{L}}$ of suprathermal particles is smaller than the width of the RDS, which is $\simeq \lambda c / 3 u_{\mathrm{s}}$ for a shock velocity $u_{\mathrm{s}}$ and photon mean free path $\lambda$. On the other hand, $r_{\mathrm{L}}$ is larger than the width of the CS where diffusive shock acceleration should become possible. A thorough understanding of the CS formation time is then crucial to study the onset of cosmic-ray (CR) acceleration, when very high energies may be reached. Indeed, supernovae in dense winds have been suggested to be the long-sought PeVatrons, see in particular References [14] and [15].

We confirm in this work that a CS forms around SB, and we further demonstrate that the CS can form significantly before SB for some progenitors enshrouded in optically thick winds. This redefines the onset of CR acceleration with respect to SB, since it can start in such cases significantly before SB. See also Reference [16] for more details. We then calculate the maximum $\mathrm{CR}$ energy that can be reached and show that CRs with energies in excess of $\sim(1-10) \mathrm{TeV}$ are expected to be produced. In Section 2, we study the formation time of the CS, analytically and numerically, both for optically thin and optically thick winds. In Section 3, we investigate particle acceleration in thick winds, and discuss in Section 4 the relating production of secondary $\sim \mathrm{TeV}$ neutrinos.

\section{Formation of a collisionless shock around supernova shock breakout}

In the following, we assume a non-relativistic shock in spherical 1D geometry with radius $r$, where $r=0$ corresponds to the centre of the progenitor. The hydrostatic core and the wind are assumed to be fully ionized hydrogen. Assuming more realistic compositions would not change our findings. For the temperatures we consider, between $\sim \mathrm{eV}$ and $\sim m_{\mathrm{e}} c^{2}$, the opacity $\kappa$ is dominated by Thomson scattering: $\kappa=\sigma_{\mathrm{t}} / m_{\mathrm{p}}$, where $\sigma_{\mathrm{t}}$ is the Thomson cross section.

\subsection{Optically thin winds}

A CS is expected to form following SB. In Lagrangian coordinates, the acceleration of a shell of wind is $D u / D t=\kappa \mathscr{F}_{\text {rad }} / c-(1 / \rho) \partial p / \partial r$, where $\mathscr{F}_{\text {rad }}$ is the photon flux, $u$ the shell velocity and $p$ the fluid pressure. The maximum velocity that can be reached by a shell, initially at $r_{\mathrm{i}}$, due to the flash of photons from breakout at $t_{\mathrm{br}}$ is $u_{\mathrm{max}, \gamma}=\kappa \int_{t_{\mathrm{br}}}^{\infty} \mathscr{F}_{\mathrm{rad}} d t / c<\kappa \int_{t_{\mathrm{br}}}^{\infty} \mathscr{L} d t / 4 \pi c r_{\mathrm{i}}^{2} \propto r_{\mathrm{i}}^{-2}$ where $\mathscr{L}$ denotes the SN luminosity. After beginning of SB at a radius $r_{\mathrm{br}}$, the formation of a collisionless shock is not immediate, see e.g. [12]. However, the $r^{-2}$ dilution of breakout photons ensures that a shell $\mathscr{S}_{1}$ initially at $r_{1} \geq r_{\text {br }}$ will catch up supersonically a shell $\mathscr{S}_{2}$ initially at $r_{2}$, with $r_{2}$ (sufficiently) larger than $r_{1}$. Despite the wind being nearly collisionless, $\mathscr{S}_{1}$ is prevented from going through $\mathscr{S}_{2}$ by electromagnetic instabilities, which gives rise to a CS (see [13] for an estimate of their growth time). For an optically thin wind, we confirm numerically the formation of a CS after SB (as found by [12] and [2]) with our code, described in Section 2.2. 


\subsection{Optically thick winds}

SB starts in the wind at $\tau \sim c / u_{\mathrm{s}}=\beta_{\mathrm{s}}^{-1}$ [4]. We have demonstrated in Ref. [16] that in the case of moderately optically thick winds, the CS actually forms before SB: The RDS stalls in the $\tau>\beta_{\mathrm{s}}^{-1}$ part of the wind, soon after exiting the hydrostatic core of the progenitor. This happens when photon dilution due to shock curvature cannot be compensated by photon creation. We have shown that, in a wind with density $\rho(r) \propto r^{-2}$, this happen if the shock velocity $u_{\mathrm{s}}$ does not exceed:

$$
u_{\mathrm{s}} \lesssim 0.1 c\left(\frac{u_{\mathrm{w}}}{10 \mathrm{~km} / \mathrm{s}}\right)\left(\frac{r_{*}}{10^{13} \mathrm{~cm}}\right)\left(\frac{\dot{M}}{5 \cdot 10^{-4} \mathrm{M}_{\odot} / \mathrm{yr}}\right)^{-1},
$$

where $u_{\mathrm{w}}$ is the wind velocity, $r_{*}$ the radius of the star, and $\dot{M}$ the mass-loss rate. Red supergiants or Wolf-Rayet stars with relatively high mass-loss rates prior to the explosion are good candidates for satisfying condition (2.1). For example, Type Ibc supernova SN 2008D/XRF 080109 may have been an event in which a CS was formed before SB: Ref. [17] suggested that SN 2008D is consistent with the explosion of a Wolf-Rayet star in a moderately thick wind, and that the progenitor underwent a steady but enhanced mass-loss during the last $\lesssim 10$ days. Interestingly, the parameters derived by [17] for SN $2008 \mathrm{D}\left(\beta_{1} \approx 0.25, u_{\mathrm{w}} \approx 1000 \mathrm{~km} \mathrm{~s}^{-1}\right.$, and $\dot{M} \approx 2 \cdot 10^{-4} \mathrm{M}_{\odot} \mathrm{yr}^{-1}$ close to the star) marginally satisfy Inequality (2.1) for $r_{*} \approx 10^{11} \mathrm{~cm}$.

In contrast, progenitors of Type IIn supernovae are not expected to satisfy this scenario, since Eq. (2.1), with typical values of $\dot{M}$ and $u_{\mathrm{w}}$ for Type IIn, implies upper limits on $u_{\mathrm{s}}$ well below the actual shock velocities. For progenitors with large $\dot{M}$, the RDS should always survive the transition from the core to the optically thick wind. For example, Type IIn supernova SN 2010j1 does not satisfy (2.1) because of the large mass-loss rate $\dot{M} \sim 1 \mathrm{M}_{\odot} \mathrm{yr}^{-1}$.

We confirm numerically the above statements with our Eulerian 1D-spherical radiation hydrodynamics code. The fluid is assumed to be fully ionized. The code is two-temperature (i.e. proton and electron temperatures are assumed to be equal). We use a gray frequency average for the radiation, and represent it by its internal energy $E_{\text {rad }}$ with characteristic temperature $T_{\text {rad }}=$ $\left(c E_{\mathrm{rad}} / 4 \sigma\right)^{1 / 4}$, where $\sigma$ is the Stefan-Boltzmann constant. At each time step, the radiation transport is solved using a "square-root" flux-limited diffusion approximation, and the opacity $\kappa$ is assumed to be dominated by Thomson scattering. For the transfer of energy between fluid and radiation, we take into account Compton cooling and bremsstrahlung, using the formulas of [12].

Our main result does not strongly depend on the density and temperature profile of the hydrostatic core, and we take as an example those for the red supergiant used in [12]. We choose initial conditions such that the shock velocity reaches $\beta_{\mathrm{s}} \approx 0.1$. We use winds with density profiles $\propto r^{-2}$. We take here $\dot{M}=5 \cdot 10^{-4} \mathrm{M}_{\odot} \mathrm{yr}^{-1}$ and $u_{\mathrm{w}}=10 \mathrm{~km} \mathrm{~s}^{-1}$. A CS appears before SB, at $r<1.5 r_{*}$. At the CS formation time, photons still have not started to escape from the optically thick material, contrary to e.g. expectations for breakout from a stellar surface. Fig. 1 shows the CS at $r \approx 1.6 r_{*}$, near the downstream of the radiation-dominated transition (remains of the initial RDS). See caption of Fig. 1 for details. It appears as a growing discontinuity in the smoother velocity profile. The radiation-dominated transition extends to radii larger than shown in Fig. 1. At such an early time, the CS downstream temperature is only $\sim 1 \mathrm{keV}$ because the radiation still provides most of the fluid acceleration in its upstream, but we find the discontinuity in $u$ to grow and the CS processes a significantly larger fraction of $\rho u_{\mathrm{s}}^{2}$ at larger $r$. For other parameter values (smaller $\beta_{\mathrm{s}}$ for $\dot{M}, u_{\mathrm{w}}$ 

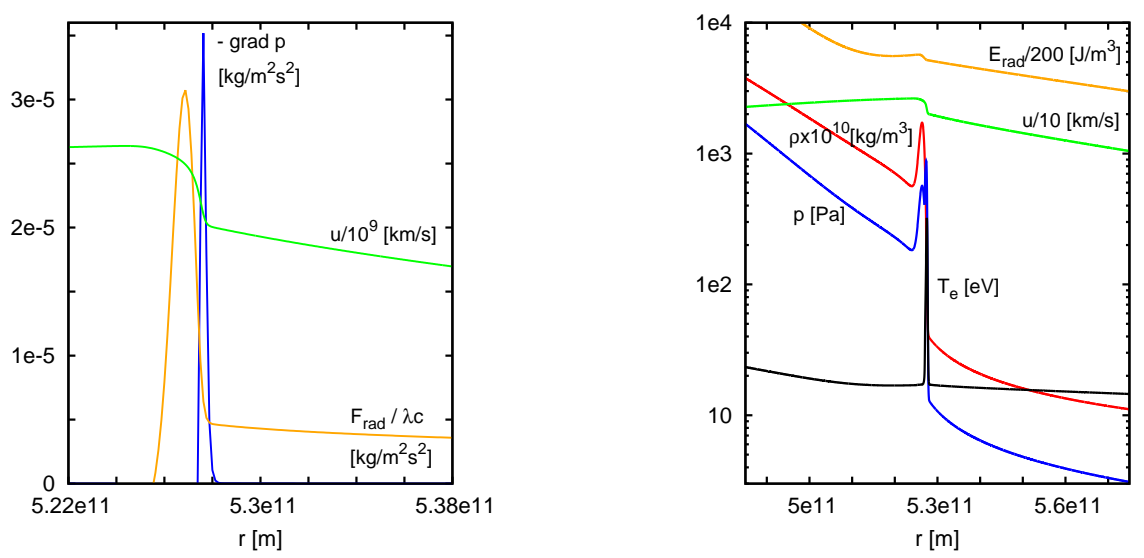

Figure 1: Simulation of a red supergiant explosion in a thick wind before breakout. Zoom around the downstream of the radiation-dominated transition of the shock, where a discontinuity (CS) can be seen around $r \approx 5.28 \times 10^{11} \mathrm{~m} \approx 1.6 r_{*} \approx r_{\mathrm{br}} / 2$. Left panel: Most of the fluid acceleration $d(\rho u) / d t$ is due to the radiation, except in a thin zone at the CS, where the fluid contribution dominates. Right panel: Peaks in $p$ and in the electron temperature $T_{\mathrm{e}}$ appear in the CS immediate downstream. $T_{\text {rad }}$ follows $T_{\mathrm{e}}$ except in the peak region.

fixed), radiation plays a smaller role, allowing the CS to emit in hard X-rays ( $\gtrsim 10 \mathrm{~s} \mathrm{keV}$ ) before its photons break out. We find that for significantly larger $\dot{M}=(1,5) \cdot 10^{-3} \mathrm{M}_{\odot} \mathrm{yr}^{-1}$ and the same core profile, the RDS survives the transition to the wind. Details of the hydrostatic core are not found to be very important, and we test the Wolf-Rayet case by rescaling, as a first approximation, the above profile to $r_{*}=10^{11} \mathrm{~cm}$ and larger densities. We vary $u_{\mathrm{s}}$ by slightly changing the explosion energy. For $\dot{M}=5 \cdot 10^{-4} \mathrm{M}_{\odot} \mathrm{yr}^{-1}$ and $u_{\mathrm{w}}=1000 \mathrm{~km} \mathrm{~s}^{-1}$, we find that for $u_{\mathrm{s}} \gtrsim 0.15 c$, no CS appears before $\mathrm{SB}$, whereas they do appear before $\mathrm{SB}$ for $u_{\mathrm{s}} \lesssim 0.1 c$.

\section{Particle acceleration}

Assuming conservatively a magnetic field strength at the CS similar to that at the stellar surface, $B_{\mathrm{S}} \sim 10 \mathrm{G}$ [18], and wind densities $\rho \sim 10^{-11(-9)} \mathrm{g} \mathrm{cm}^{-3}$, the CS is super-Alfvénic. Once it is formed, CR acceleration may start. Coulomb losses for suprathermal particles are sufficiently small here and do not prevent them from entering diffusive shock acceleration and being accelerated. However, for Wolf-Rayet stars with $\dot{M} \gtrsim 10^{-3} \mathrm{M}_{\odot} \mathrm{yr}^{-1}$, such losses start to inhibit CR acceleration before SB. Some findings of [13] and [19] can be transposed to our study, yet we deal here with a shock propagating in denser regions of the wind. Assuming Bohm diffusion for CRs at the CS [20,21], and equal dwell times in the downstream and the upstream, one finds a typical acceleration time

$$
\tau_{\mathrm{CR}} \approx \frac{8 E_{\mathrm{CR}}}{3 e B_{\mathrm{s}} u_{\mathrm{s}}^{2}} \approx 30 \mathrm{~s}\left(\frac{E_{\mathrm{CR}}}{10 \mathrm{TeV}}\right)\left(\frac{B_{\mathrm{s}}}{10 \mathrm{G}}\right)^{-1}\left(\frac{\beta_{\mathrm{s}}}{0.1}\right)^{-2}
$$

for protons with energy $E_{\mathrm{CR}}$. This time can be optimistic when the discontinuity in velocity at the shock is still small due to smoothing by radiation. However, magnetic field amplification at 
the shock due to the non-resonant hybrid (NRH) instability [22] plays a role in the opposite direction by diminishing $\tau_{\mathrm{CR}}$ and thereby facilitating CR acceleration. Magnetic field amplification is (constantly) driven by the escape of the highest energy CRs in the upstream of the collisionless shock, see [15]. For the ranges of parameter values that are relevant here, the typical growth time of the NRH instability is smaller than the damping time of the turbulence by the radiation field, which energy density is $U_{\text {rad }} \approx \rho u_{\mathrm{s}}^{2}$. Therefore, magnetic field amplification should occur in such conditions. In the upstream of the CS, a turbulent fluid parcel with velocity $u_{\mathrm{t}}$ suffers momentum losses due to radiation (second order Fermi for photons). From the momentum equation of the fluid parcel, one can deduce the typical damping time of the turbulence :

$$
\tau_{\mathrm{damp}}=\frac{u_{\mathrm{t}}}{d u_{\mathrm{t}} / d t} \approx \frac{c^{2}}{\kappa \rho u_{\mathrm{s}}^{2} u_{\mathrm{t}}} \gtrsim \frac{c^{2}}{\kappa \rho u_{\mathrm{s}}^{3}} .
$$

The size of the discontinuity in velocity at the CS may be written as $\Delta u=\frac{3}{4} f u_{\mathrm{s}}$, where $0<f \leq 1$ and $f=1$ is the limiting case where no radiation accelerates the upstream of the CS. The growth rate of the fastest growing mode of the NRH instability is equal to $\gamma_{\max }=0.5 j_{\mathrm{CR}} \sqrt{\mu_{0} / \rho}$, where $j_{\mathrm{CR}} \simeq 0.03 \rho f^{2} u_{\mathrm{s}}^{3} e / E_{\mathrm{CR}}$ is the CR current density which drives it [15]. The instability growth time, $\tau_{\mathrm{NRH}} \approx 5 \gamma_{\max }^{-1}$, is then small compared to $\tau_{\mathrm{damp}}:$

$$
\begin{aligned}
& \frac{\tau_{\mathrm{NRH}}}{\tau_{\mathrm{damp}}} \lesssim \frac{10 \kappa \sqrt{\rho} E_{\mathrm{CR}}}{0.03 c^{2} e \sqrt{\mu_{0}} f^{2}} \simeq 0.08\left(\frac{E_{\mathrm{CR}}}{10 \mathrm{TeV}}\right)\left(\frac{f}{0.05}\right)^{-2} \\
& \times\left(\frac{\dot{M}}{5 \cdot 10^{-4} \mathrm{M}_{\odot} / \mathrm{yr}}\right)^{1 / 2}\left(\frac{u_{\mathrm{w}}}{10 \mathrm{~km} / \mathrm{s}}\right)^{-1 / 2}\left(\frac{r}{10^{13} \mathrm{~cm}}\right)^{-1}
\end{aligned}
$$

numerically for a wind with $\rho \propto r^{-2}$.

Let us note that $10 \mathrm{TeV}$ energies are reachable before breakout because $\tau_{\mathrm{CR}} \ll\left(r_{\mathrm{br}}-r_{*}\right) / u_{\mathrm{s}} \approx$ several hours (resp. minutes) for red supergiant (resp. Wolf-Rayet) progenitors with $\beta_{\mathrm{s}}=0.1$ and a shock breakout radius $r_{\mathrm{br}} \approx 10 r_{*}$. For such red supergiants, $\tau_{\mathrm{CR}}($ at $10 \mathrm{TeV})$ is smaller than energy loss times from pion production through inelastic $p p$ and $p \gamma$ collisions. The typical life time of a CR proton due to $p p$ collisions, $\tau_{\mathrm{pp}} \simeq m_{\mathrm{p}} / 0.2 c \rho \sigma_{\mathrm{pp}}$, is

$$
\tau_{\mathrm{pp}} \approx 4 \min \left(\frac{u_{\mathrm{w}}}{10 \mathrm{~km} / \mathrm{s}}\right)\left(\frac{r}{10^{13} \mathrm{~cm}}\right)^{2}\left(\frac{\dot{M}}{5 \cdot 10^{-4} \mathrm{M}_{\odot} / \mathrm{yr}}\right)^{-1}
$$

The background $\sim 10 \mathrm{eV}$ photons in the thick wind are not sufficiently energetic to trigger pion production through inelastic $p \gamma$ scattering. For $10 \mathrm{TeV}$ CRs, $\gtrsim 10 \mathrm{keV}$ photons are required to exceed the threshold for pion production. Photons with such energies can be produced by the radiative CS. However, the number density of target photons $n_{\gamma}$ must be much less than $\rho u_{\mathrm{s}}^{2} / h v$ [19]. We find for the typical life time of a CR proton due to $p \gamma$ collisions, $\tau_{\mathrm{p} \gamma} \simeq 1 / 0.2 c n_{\gamma} \sigma_{\mathrm{p} \gamma}$ :

$$
\tau_{\mathrm{p} \gamma} \gtrsim 2 \min \left(\frac{u_{\mathrm{w}}}{10 \mathrm{~km} / \mathrm{s}}\right)\left(\frac{r}{10^{13} \mathrm{~cm}}\right)^{2}\left(\frac{\dot{M}}{5 \cdot 10^{-4} \mathrm{M}_{\odot} / \mathrm{yr}}\right)^{-1}\left(\frac{u_{\mathrm{s}}}{0.1 c}\right)^{-2}\left(\frac{E_{\mathrm{CR}}}{10 \mathrm{TeV}}\right)^{-1}
$$

$e^{ \pm}$pair creation due to $p \gamma$ interactions does not yield a stronger constraint.

In the case of Wolf-Rayet progenitors with the above parameters, $\tau_{\mathrm{pp}, \mathrm{p} \gamma} \gtrsim 3 \mathrm{~s}$. Consequently, $\mathrm{TeV}$ energies may be reached for Wolf-Rayet stars. 


\section{Secondary TeV neutrinos}

Our findings provide a new technique to access information on SN progenitors inside thick winds, such as the radius of the stellar core $r_{*}$, and the density profile at $\tau \gtrsim \beta_{\mathrm{s}}^{-1}$. Indeed, the presence of $\gtrsim(1-10) \mathrm{TeV}$ CRs in such dense circumstellar winds would lead to the creation of secondary $\gtrsim 100 \mathrm{GeV}-1 \mathrm{TeV}$ neutrinos (from notably $\pi^{ \pm}$decay).

By detecting such secondary neutrinos before the first photons from breakout, one will improve our knowledge of the still poorly understood late stages of massive star evolution. The time interval between the arrival of the first neutrinos and photons is $\Delta t_{v \gamma} \approx\left(r_{\mathrm{br}}-r_{*}\right)\left(\beta_{\mathrm{s}}^{-1}-1\right) / c \approx 8 \mathrm{hr}$ (resp. $5 \mathrm{~min}$ ) for red supergiants (resp. Wolf-Rayets) with the above parameters, $u_{\mathrm{s}}=0.1 \mathrm{c}$, and a shock breakout radius $r_{\mathrm{br}}=10 r_{*}$. Assuming that $5 \%$ of the energy processed by the shock is channelled into CRs, we typically find for a source at distance $l$, and a processed mass between $r_{*}$ and $r_{\mathrm{br}}$ of $\approx 10^{-5} \mathrm{M}_{\odot}$, that $\sim 10^{3}(3 \mathrm{kpc} / l)^{2}$ neutrinos with $\sim \mathrm{TeV}$ energies would be detectable before $\mathrm{SB}$ by IceCube or KM3NeT. One could record a few of such neutrinos for an event in the Magellanic Clouds.

\section{Conclusions}

In a core-collapse supernova, a RDS propagates through its progenitor star. If the circumstellar wind is optically thin, the RDS stalls when it reaches the outer layers of the stellar core and a collisionless shock forms in the wind. For progenitors with optically thick winds, the formation of a CS should also occur no later than during or on the time scale of shock breakout. However, we have pointed out in Section 2.2 that for some astrophysically-relevant progenitors surrounded with thick winds, a CS forms well before SB. In such cases, the RDS stalls when entering the optically thick part of the wind, notably because of shock curvature. Wolf-Rayet stars or red supergiants with either dense winds or enhanced mass-loss prior to the explosion are good candidates. On the other hand, Type IIn supernovae are expected to have too dense winds to form CS when the RDS leave their cores.

In Section 3, we have discussed the onset of particle acceleration at the CS, in the case where the CS is formed before SB from an optically thick wind. We have calculated the maximum CR energy that can be reached in such environments. We have found that protons are rapidly accelerated to multi-TeV energies before SB, both for Wolf-Rayet and red supergiant progenitors. After SB, significantly higher energies are expected to be reached in such dense winds, see Reference [15].

Finally, in Section 4, we have discussed the production of secondary $\sim \mathrm{TeV}$ neutrinos from the destruction of these CRs via $p p$ and $p \gamma$ interactions, as well as the prospects for detecting these neutrinos.

\section{References}

[1] S. A. Colgate, ApJ 187 (1974) 321.

[2] L. Ensman, A. Burrows, Shock breakout in SN 1987A, ApJ 393 (1992) 742.

[3] S. Blinnikov, P. Lundqvist, O. Bartunov, K. 'i. Nomoto, K. Iwamoto, Radiation hydrodynamics of sn 1987a: I. global analysis of the light curve for the first 4 months, ApJ 532 (2000) 1132 [astro-ph/9911205]. 
[4] B. Katz, R. Budnik, E. Waxman, Fast radiation mediated shocks and supernova shock breakouts, ApJ 716 (2010) 781 [arXiv:0902.4708].

[5] E. Nakar, R. 'e. Sari, Early supernovae light-curves following the shock-breakout, ApJ 725 (2010) 904 [arXiv:1004.2496].

[6] N. Sapir, B. Katz, E. Waxman, Non-relativistic radiation mediated shock breakouts: I. Exact bolometric planar breakout solutions, ApJ 742 (2011) 36 [arXiv:1103.5075].

[7] S. Campana, V. Mangano, A. J. Blustin, P. Brown, D. N. Burrows, G. Chincarini, J. R. Cummings, G. Cusumano et al., The shock break-out of grb 060218/sn 2006aj, Nature 442 (2006) 1008 [astro-ph/0603279].

[8] S. Gezari, L. Dessart, S. Basa, D. C. Martin, J. D. Neill, S. E. Woosley, D. J. Hillier, P. Astier et al., Probing Shock Breakout with Serendipitous GALEX Detections of Two SNLS Type II-P Supernovae, ApJ 683 (2008) L131 [arXiv:0804.1123].

[9] K. Schawinski, S. Justham, C. Wolf, P. Podsiadlowski, M. Sullivan, K. C. Steenbrugge, T. Bell, H. -J. Roeser et al., Supernova shock breakout from a red supergiant, Science 321 (2008) 223 [arXiv:0803.3596].

[10] M. Modjaz, W. Li, N. Butler, R. Chornock, D. Perley, S. Blondin, J. S. Bloom, A. V. Filippenko et al., From shock breakout to peak and beyond: Extensive panchromatic observations of the aspherical type Ib supernova $2008 D$ associated with Swift X-ray transient 080109, ApJ 702 (2009) 226 [arXiv:0805.2201].

[11] E. O. Ofek, I. Rabinak, J. D. Neill, I. Arcavi, S. B. Cenko, E. Waxman, S. R. Kulkarni, A. G. Yam et al., Supernova PTF 09uj: A possible shock breakout from a dense circumstellar wind, ApJ 724 (2010) 1396 [arXiv:1009.5378].

[12] R. A. Chevalier, R. I. Klein, Nonequilibrium processes in the evolution of type II supernovae, ApJ 234 (1979) 597.

[13] E. Waxman and A. Loeb, TeV neutrinos and GeV photons from shock breakout in supernovae, Phys. Rev. Lett. 87 (2001) 071101 [astro-ph/0102317].

[14] V. Tatischeff, Radio emission and nonlinear diffusive shock acceleration of cosmic rays in the supernova SN 1993J, Astron. Astrophys. 499 (2009) 191 [arXiv:0903.2944].

[15] A. R. Bell, K. Schure, B. Reville, G. Giacinti, Cosmic ray acceleration and escape from supernova remnants, MNRAS 431 (2013) 415 [arXiv:1301.7264].

[16] G. Giacinti, A. R. Bell, Collisionless shocks and TeV neutrinos before supernova shock breakout from an optically thick wind, MNRAS 449 (2015) 3693 [arXiv:1503.04170].

[17] G. Svirski, E. Nakar, SN 2008D: A Wolf-Rayet explosion through a thick wind, ApJ 788 (2014) L14 [arXiv:1403.3400].

[18] R. Barvainis, G. McIntoch, C. R. Predmore, Evidence for strong magnetic fields in the inner envelopes of late-type stars, Nature 329 (1987) 613.

[19] B. Katz, N. Sapir, E. Waxman, X-rays, gamma-rays and neutrinos from collisionless shocks in supernova wind breakouts, Proc. of the International Astronomical Union 7 (2011) 274 [arXiv:1106.1898].

[20] B. Reville, A. R. Bell, Universal behaviour of shock precursors in the presence of efficient cosmic-ray acceleration, MNRAS 430 (2013) 2873 [arXiv:1301.3173]. 
[21] D. Caprioli, A. Spitkovsky, Simulations of Ion Acceleration at Non-relativistic Shocks. II. Magnetic Field Amplification, ApJ 794 (2014) 46 [arXiv:1401.7679].

[22] A. R. Bell, Turbulent amplification of magnetic field and diffusive shock acceleration of cosmic rays, MNRAS 353 (2004) 550. 